\title{
Estoques de carbono e nitrogênio e fração leve da matéria orgânica em Neossolo Quartzarênico sob uso agrícola
}

\author{
Leidivan Almeida Frazão(1), Ingrid Kely da Silva Santana(2), David Vilas Boas de Campos(2), \\ Brigitte Josefine Feigl(1) e Carlos Clemente Cerri(1)
}

\begin{abstract}
(1)Universidade de São Paulo, Centro de Energia Nuclear na Agricultura, Laboratório de Biogeoquímica Ambiental, Avenida Centenário 303, Caixa Postal 96, CEP 13416-000 Piracicaba, SP. E-mail: lafrazao@cena.usp.br, beduardo@cena.usp.br, cerri@cena.usp.br (2)Embrapa Solos, Rua Jardim Botânico 1.024, CEP 22460-000 Rio de Janeiro, RJ. E-mail: ingridkelysantana@gmail.com, davidcampos@cnps.embrapa.br E-mail: leidivan_frazao@yahoo.com.br
\end{abstract}

Resumo - O objetivo deste trabalho foi avaliar os estoques de $\mathrm{C}$ e $\mathrm{N}$ do solo e os teores destes elementos na fração leve livre (FLL) da matéria orgânica (MOS) de um Neossolo Quartzarênico, sob diferentes usos agrícolas, no Cerrado. Foram selecionadas cinco áreas para avaliação: cerrado nativo; duas áreas com plantio convencional de soja; uma área ocupada anteriormente com pastagem e convertida em plantio direto de soja; e uma área com pastagem de baixa produtividade. Amostras de solo foram coletadas das camadas de 0-5, 5-10, 10-20 e 20-30 cm de profundidade, para determinação dos estoques de carbono (C) e nitrogênio (N) totais do solo. Os teores destes elementos na FLL foram determinados nas profundidades $0-5$ e 5-10 cm. Em comparação à área sob cerrado, foi observado aumento nos estoques de $\mathrm{C}$ total do solo nas áreas de soja com até cinco anos de implantação. Houve redução das quantidades de FLL e dos teores de C à profundidade 0-5 cm, nas áreas com plantio de soja e pastagem. Considerando-se o baixo aporte natural de $\mathrm{C}$ e $\mathrm{N}$ no Neossolo Quartzarênico avaliado, é necessária a adoção de sistemas agrícolas que promovam aporte adequado de resíduos para a manutenção ou incremento da matéria orgânica do solo.

Termos para indexação: Cerrado, pastagem, plantio convencional, plantio direto.

\section{Carbon and nitrogen stocks and organic matter light fraction in a Typic Quartzipsamment under agricultural use}

\begin{abstract}
The objective of this work was to evaluate $\mathrm{C}$ and N stocks in the soil and their contents in the free light fraction (FLL) of the soil organic matter (MOS), in a Typic Quartzipsamment under different land-use in Cerrado. Five areas were selected for evaluation: native cerrado; two areas with soybean under conventional tillage; one area formerly under pasture and afterwards cultivated with soybean under no-tillage; and one area under pasture with low productivity. Soil samples were collected from 0-5, 5-10, 10-20 and 20-30-cm depth layers to determine total $\mathrm{C}$ and $\mathrm{N}$ stocks of the soil. Contents of $\mathrm{C}$ and $\mathrm{N}$ in the FLL were determined at $0-5$ and $5-10-\mathrm{cm}$ depth. An increase was observed in the total C stocks in soybean areas implemented until five years, in comparison to the native cerrado area. There was a reduction in the amount of FLL and C content at $0-5 \mathrm{~cm}$ depth, in areas with soybean and pasture. Considering the low $\mathrm{C}$ and $\mathrm{N}$ natural input onto the Typic Quartzipsamment, the adoption of agricultural systems that can promote an adequate supply of residues is necessary for maintaining or increasing the soil organic matter.
\end{abstract}

Index terms: Cerrado, pasture, conventional tillage, no-tillage.

\section{Introdução}

Obioma Cerrado ocupa 24,4\% do território brasileiro. Seu desmatamento iniciou-se, principalmente, com o processo de expansão da produção agrícola a partir da década de 70. Na maioria das áreas, os solos foram primeiramente manejados com pastagem e, posteriormente, com culturas anuais.

A mudança no uso da terra, provocada pela ação humana, tem o potencial de alterar significativamente a dinâmica da matéria orgânica do solo (MOS) (Schlesinger, 1984), a qual constitui um reservatório de materiais diferentes quanto à origem, composição e dinâmica. Assim, os estoques de $\mathrm{C}$ e de $\mathrm{N}$ contidos nos solos podem ser submetidos a modificações, quando a vegetação nativa é retirada para a conversão da área em sistemas agrícolas. Conforme o sistema de manejo do solo empregado, esses estoques podem permanecer estáveis, aumentar ou diminuir em relação ao sistema natural.

Entre os diversos enfoques relacionados à caracterização da qualidade da MOS (Tiessen \& Stewart, 1983; Stevenson \& Elliot, 1989; Christensen, 1992), a análise das frações separadas fisicamente ocupa

Pesq. agropec. bras., Brasília, v.45, n.10, p.1198-1204, out. 2010 
lugar de destaque, pois há evidências de que tais frações estão associadas a funções específicas na dinâmica da MOS (Stevenson \& Elliot, 1989). Com o objetivo de identificar em quais compartimentos o carbono se acumula, têm sido utilizadas técnicas de fracionamento físico da MOS, que podem ser granulométricas (Feller \& Beare, 1997; Needelman et al., 1999), densimétricas (Golchin et al., 1994) ou uma mistura de ambas (Six et al., 1998). O fracionamento físico, baseado no tamanho (granulométrico) ou na densidade (densimétrico) das partículas do solo, é utilizado em estudos de quantificação e caracterização dos compartimentos da MOS (Cambardella \& Elliott, 1992; Roscoe \& Machado, 2002; Diekow et al., 2005).

É importante destacar que os estoques de $\mathrm{C}$ do solo estão diretamente relacionados à textura do solo, e que há uma clara tendência de aumento com a elevação dos teores de argila, o que está relacionado à proteção física e química que essa fração exerce sobre a MOS (Feller \& Beare, 1997; Roscoe \& Machado, 2002).

As frações orgânicas de menor tempo de ciclagem, que respondem mais rapidamente às mudanças do uso do solo, correspondem a uma pequena fração do teor total de C orgânico (Christensen, 1996), e os indicadores mais responsivos às alterações da qualidade do solo estão associados aos compartimentos mais lábeis da MOS como, por exemplo, o compartimento da fração leve (Stevenson, 1994). A fração leve (FL) da MOS é derivada de resíduos de plantas, raízes e hifas que ainda representam estruturas celulares reconhecíveis. Por ser sensível às práticas de manejo, a determinação da FL é importante na avaliação da qualidade do sistema de manejo em curto prazo (Gregorich et al., 2006; Jinbo et al., 2007; Rangel \& Silva, 2007). A fração leve livre (FLL) possui composição comparável àquela dos materiais vegetais (Freixo et al., 2002), e seu único mecanismo de proteção atuante é a recalcitrância molecular (Sollins et al., 1996).

O objetivo deste estudo foi quantificar os estoques de $\mathrm{C}$ e $\mathrm{N}$ do solo e os teores destes elementos, na fração leve livre da MOS de um Neossolo Quartzarênico, sob diferentes usos agrícolas no Cerrado.

\section{Material e Métodos}

O presente estudo foi realizado nas fazendas Santa

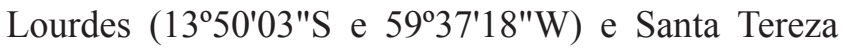
$\left(13^{\circ} 50^{\prime} 00^{\prime \prime} \mathrm{S}\right.$ e $\left.59^{\circ} 37^{\prime} 18^{\prime \prime} \mathrm{W}\right)$, localizadas no Municipio de Comodoro, MT. O solo estudado é classificado como Neossolo Quartzarênico, e as principais características físicas e químicas das áreas de estudo estão descritas na Tabela 1. Informações mais detalhadas estão disponíveis em Frazão et al. (2008).

As áreas de estudo foram selecionadas pela representatividade do histórico de uso da terra, no Estado do Mato Grosso, e pela textura do solo, visto que o Neossolo Quartzarênico foi incorporado recentemente ao processo de produção de grãos em áreas de Cerrado.

De acordo com a classificação de Köppen, o clima local é do tipo Aw (tropical chuvoso), com chuvas concentradas no verão (outubro a abril). O período seco é bem definido durante a estação de inverno (maio a setembro). A média anual da precipitação pluvial é $1.900 \mathrm{~mm}$ por ano, e a temperatura média anual é $26^{\circ} \mathrm{C}$.

Os tratamentos constituíram-se de cinco áreas de amostragem, caracterizadas da seguinte forma: cerrado (CE), solo sob vegetação nativa, utilizado neste estudo como referência para estocagem e conservação de carbono; plantio convencional (PC-1), área desmatada e posteriormente cultivada por um ano com a cultura da soja (Glycine max L.) pelo sistema plantio convencional (SPC); plantio convencional (PC-4), área desmatada e imediatamente cultivada por um ano com arroz (Oriza sativa L.) e, em seguida, por mais três anos com a cultura da soja em sucessão a culturas com milheto (Pennisetum glaucum L.) sob SPC; pastagem convertida em plantio direto (PA-13/PD-5), área desmatada e cultivada com arroz pelo SPC por um ano, depois com pastagem por treze anos, sem reforma e, posteriormente, convertida ao sistema plantio direto (SPD), com o cultivo da soja em sucessão a culturas com milheto por cinco anos; pastagem (PA-22), área desmatada e cultivada por um ano com arroz pelo SPC e vinte e dois anos com pastagem de braquiária [Urochloa decumbens (Stapf.) Webster], tendo permanecido sem reforma, e caracterizada como de baixa produtividade no momento da amostragem.

As áreas CE, PC-1 e PA-13/PD-5 localizam-se na fazenda Santa Lourdes, e as áreas PC-4 e PA-22, na fazenda Santa Tereza. A altitude das áreas de estudo estão em torno de $600 \mathrm{~m}$, e a distância entre as duas fazendas é de $4 \mathrm{~km}$.

Para corrigir a acidez do solo, após o desmatamento e a queima da vegetação remanescente do cerrado, aplicaram-se $3 \mathrm{Mg}$ ha $^{-1}$ de calcário dolomítico ao 
solo em todas as áreas de estudo. Nas áreas com a cultura de soja, foi empregada a adubação de plantio com $400 \mathrm{~kg} \mathrm{ha}^{-1}$ de NPK com formulação 2-24-12 e, em cobertura, com $100 \mathrm{~kg} \mathrm{ha}^{-1}$ de $\mathrm{KCl}$, em área total. Para o cultivo do milheto, na área PC-4, aplicaram-se $200 \mathrm{~kg} \mathrm{ha}^{-1}$ de NPK a 2-24-12. Na área cultivada com pastagem (PA-22), não houve aplicação de fertilizantes.

A amostragem de solo foi realizada em julho de 2005. Para cada uma das áreas selecionadas, foi delimitada uma parcela de $10.000 \mathrm{~m}^{2}$, onde foram abertas cinco trincheiras, uma central e quatro adjacentes, com distância de $50 \mathrm{~m}$ entre elas. De cada parcela, foram coletadas cinco amostras compostas, das camadas $0-5$, 5-10, 10-20 e 20-30 cm de profundidade. As amostras utilizadas para a análise dos teores de $\mathrm{C}$ e $\mathrm{N}$ total foram secas ao ar, homogeneizadas, moídas, passadas por peneiras de $0,150 \mathrm{~mm}$ e analisadas por combustão a seco no equipamento Carbo Erba CHN 1110 (Carbo Erba, Milan, Itália).

A densidade do solo, utilizada para o cálculo dos estoques de $\mathrm{C}$ e $\mathrm{N}$, foi determinada em amostras indeformadas, coletadas com anéis de aço inox com $5 \mathrm{~cm}$ de diâmetro e de altura, conforme Blake \& Hartge (1986). O cálculo dos estoques foi realizado pela seguinte equação: $\mathrm{E}=\mu \mathrm{xh} \times(\mathrm{C}$ ou $\mathrm{N})$, em que: E é o estoque de carbono ou de nitrogênio $\left(\mathrm{Mg} \mathrm{ha}^{-1}\right)$; $\mu$ é a densidade do solo $\left(\mathrm{g} \mathrm{cm}^{-3}\right)$; h é a espessura da camada de solo amostrada $(\mathrm{cm}) ; \mathrm{C}$ ou $\mathrm{N}$ é o teor de $\mathrm{C}$ ou $\mathrm{N}$ do solo (\%).

Os estoques de $\mathrm{C}$ e $\mathrm{N}$ foram corrigidos para uma profundidade equivalente, isto é, as diferentes áreas em uso tiveram a massa de solo existente em determinada área $\left(10.000 \mathrm{~m}^{2}\right)$ e profundidade $(0-30 \mathrm{~cm})$ corrigidas para a massa de solo correspondente à do cerrado nativo (utilizado como área de referência). Os estoques foram calculados, tendo-se subtraido o conteúdo de $\mathrm{C}$ e $\mathrm{N}$ da massa extra de solo da camada mais profunda (20-30 cm) (Ellert \& Bettany, 1995).

A fração leve livre foi quantificada nas camadas 0-5 e 5-10 cm de profundidade, com três repetições, conforme Sohi et al. (2001), modificado por Machado (2002). Os teores de C e $\mathrm{N}$ foram determinados por combustão a seco, em amostras previamente maceradas em almorafiz, com auxílio de analisador elementar Perkin-Elmer CHNS/O 2400 (Perkin Elmer, Boston, EUA). Posteriormente, foram calculados os estoques de $\mathrm{C}$ e $\mathrm{N}$ da fração leve livre $\left(\mathrm{Mg} \mathrm{ha}^{-1}\right)$.

Para verificar a existência de diferenças significativas nos estoques de $\mathrm{C}$ e $\mathrm{N}$ da fração leve da MOS entre os tratamentos avaliados, foi aplicado o teste $\mathrm{F}$ da análise de variância. Para os dados analisados, cujo teste $\mathrm{F}$ foi significativo, as comparações de médias foram realizadas pelo teste de Tukey a 5\% de probabilidade.

\section{Resultados e Discussão}

Os estoques de $\mathrm{C}$ total do solo variaram entre $15,8 \mathrm{e}$ $28,1 \mathrm{Mg} \mathrm{ha}^{-1}$, e os estoques de $\mathrm{N}$ entre 1,0 e 1,8 $\mathrm{Mg} \mathrm{ha}^{-1}$ para a profundidade $0-30 \mathrm{~cm}$ (Figura 1). Esses valores estão dentro da faixa citada na literatura, para solos arenosos sob diferentes coberturas vegetais e biomas brasileiros (Zinn et al., 2002; Bochner et al., 2008), e confirmam as observações realizadas em estudos sobre

Tabela 1. Características físicas e químicas do solo $(0-10 \mathrm{~cm})$, em áreas de diferentes usos da terra ${ }^{(1)}$, localizadas no Municipio de Comodoro, MT.

\begin{tabular}{|c|c|c|c|c|c|}
\hline \multirow[t]{2}{*}{ Atributos } & \multicolumn{3}{|c|}{ Fazenda Santa Lourdes } & \multicolumn{2}{|c|}{ Fazenda Santa Tereza } \\
\hline & $\mathrm{CE}$ & PC-1 & PA-13/PD-5 & PC-4 & PA-22 \\
\hline Densidade do solo $\left(\mathrm{g} \mathrm{cm}^{-3}\right)$ & 1,3 & 1,3 & 1,3 & 1,3 & 1,3 \\
\hline Teor de argila $\left(\mathrm{g} \mathrm{kg}^{-1}\right)$ & 70 & 55 & 80 & 110 & 45 \\
\hline Teor de silte $\left(\mathrm{g} \mathrm{kg}^{-1}\right)$ & 20 & 15 & 20 & 20 & 15 \\
\hline Teor de areia $\left(\mathrm{g} \mathrm{kg}^{-1}\right)$ & 910 & 930 & 900 & 870 & 940 \\
\hline $\mathrm{pH} \mathrm{H} \mathrm{H}_{2} \mathrm{O}$ & 6,0 & 6,8 & 6,8 & 6,3 & 6,5 \\
\hline $\mathrm{H}+\mathrm{Al}\left(\mathrm{mmol}_{\mathrm{c}} \mathrm{dm}^{-3}\right)$ & 41,0 & 14,1 & 14,3 & 22,6 & 24,6 \\
\hline $\mathrm{Al}^{3+}\left(\mathrm{mmol}_{\mathrm{c}} \mathrm{dm}^{-3}\right)$ & 11,0 & 0,5 & 0,5 & 0,7 & 4,1 \\
\hline $\mathrm{CTC}\left(\mathrm{mmol}_{\mathrm{c}} \mathrm{dm}^{-3}\right)$ & 43,4 & 37,1 & 37,3 & 40,7 & 27,9 \\
\hline $\mathrm{K}^{+}\left(\mathrm{mmol}_{\mathrm{c}} \mathrm{dm}^{-3}\right)$ & 0,4 & 0,6 & 0,7 & 0,6 & 0,3 \\
\hline $\mathrm{Ca}^{2+}\left(\mathrm{mmol}_{\mathrm{c}} \mathrm{dm}^{-3}\right)$ & 1,0 & 19,0 & 17,6 & 9,8 & 1,7 \\
\hline $\mathrm{Mg}^{2+}\left(\mathrm{mmol}_{\mathrm{c}} \mathrm{dm}^{-3}\right)$ & 1,0 & 3,4 & 4,8 & 7,7 & 1,3 \\
\hline $\mathrm{V}(\%)$ & 5,7 & 61,4 & 61,8 & 44,8 & 11,7 \\
\hline
\end{tabular}

${ }^{(1)} \mathrm{CE}$, cerrado nativo; PC-1, soja por 1 ano (SPC); PC-4, soja por 3 anos (SPC); PA-13/PD-5, pastagem por 13 anos seguida de soja 5 por anos (SPD); PA-22, pastagem por 22 anos. 
a limitação de solos arenosos quanto à manutenção ou incremento da MOS, em razão do tamanho das partículas e da frágil estrutura física desses solos (Silva et al., 1994; Hassink, 1997; Zinn et al., 2005; Garcia-Pausas et al., 2007)

$\mathrm{O}$ incremento nos estoques de $\mathrm{C}$ e $\mathrm{N}$ do solo sob PC-1 ocorreu pela incorporação dos resíduos vegetais remanescentes, nas camadas abaixo de $10 \mathrm{~cm}$ de profundidade. Após a retirada da vegetação nativa (cerrado), o preparo do solo com a utilização do arado promoveu essa incorporação. Pereira et al. (2010) encontrou resultado semelhante no sistema preparo convencional com revolvimento do solo.

O sistema PC-4 apresentou os maiores estoques de $\mathrm{C}$ e $\mathrm{N}$, independentemente da camada amostrada, resultado que pode ser atribuído aos teores de argila um pouco mais elevados nessa área (Roscoe \& Machado, 2002), e ao recente histórico de uso deste solo. Esses resultados indicam que a incorporação de resíduos abaixo de $10 \mathrm{~cm}$ de profundidade, no preparo do solo, e

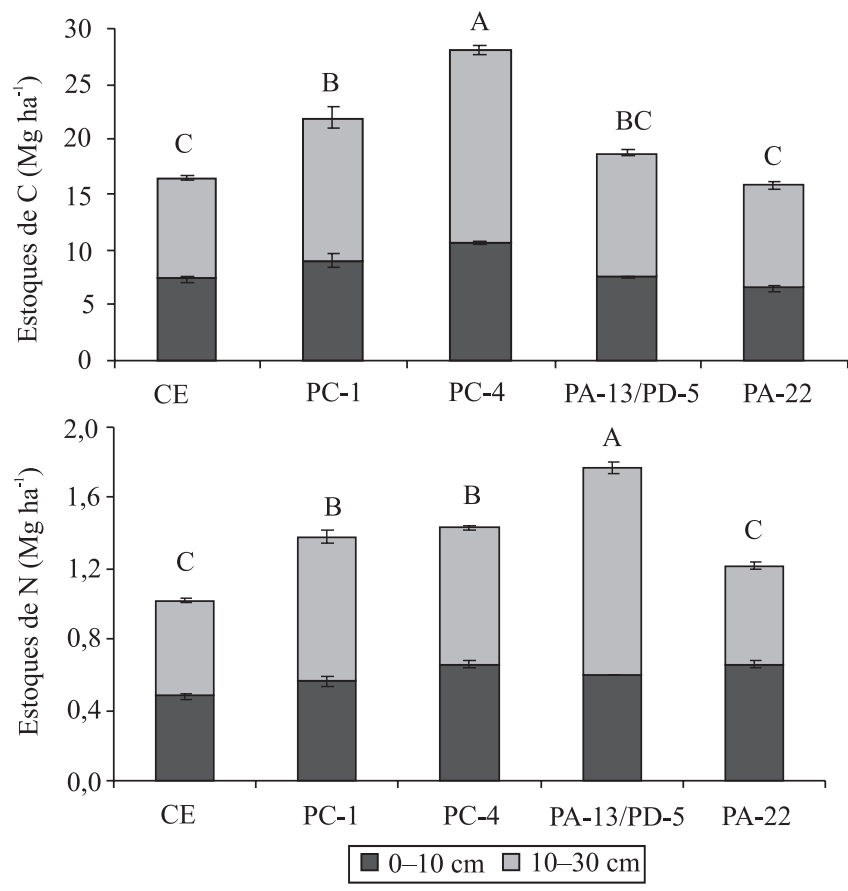

Figura 1. Estoques de carbono $(\mathrm{C})$ e nitrogênio $(\mathrm{N})$ nas camadas de 0-10 e 10-30 cm, de um Neossolo Quartzarênico sob diferentes usos agrícolas. Médias \pm erro-padrão seguidas por letras iguais não diferem entre si, pelo teste de Tukey, a $5 \%$ de probabilidade. $\mathrm{CE}$, cerrado nativo; $\mathrm{PC}-1$, soja por 1 ano (SPC); PC-4, soja por 3 anos (SPC); PA-13/PD-5, pastagem por 13 anos seguida de soja por 5 anos (SPD); PA22 , pastagem por 22 anos. a aplicação de corretivos e fertilizantes são fatores que mantêm ou incrementam a fertilidade e a MOS dessas áreas nos primeiros anos de implantação.

Antes da implantação do plantio direto, o solo do tratamento PA-13/PD-5 encontrava-se sob pastagem degradada, com reduzidos estoques de C e N. Estudos em que se compararam solos do Cerrado - com diferentes texturas - detectaram que as maiores perdas carbono orgânico do solo se relacionaram a solos mais arenosos e a frações mais leves da matéria orgânica (Silva et al., 1994; Zinn et al., 2002). Contudo, após 5 anos da implantação de plantio direto, sem o revolvimento do solo, os estoques de $\mathrm{C}$ ficaram próximos aos observados sob vegetação nativa, o que indica que houve pequeno incremento deste elemento ao solo.

$\mathrm{Na}$ área ocupada por 22 anos com pastagem (PA-22), os estoques de $\mathrm{C}$ e $\mathrm{N}$ foram próximos aos observados na área nativa. A ausência de aplicação de fertilizantes e corretivos ao solo sob pastagem justifica esses valores, visto que o manejo adequado de áreas com pastagem tende a aumentar os estoques de $\mathrm{C}$ e $\mathrm{N}$ no solo (Trumbore et al., 1995; Moraes et al., 2002; Silva et al., 2004).

De acordo com o observado, em Neossolos degradados, sob uso intensivo na região do Cerrado, a recuperação da MOS deverá ser lenta, mesmo adotando-se o sistema plantio direto e adubações

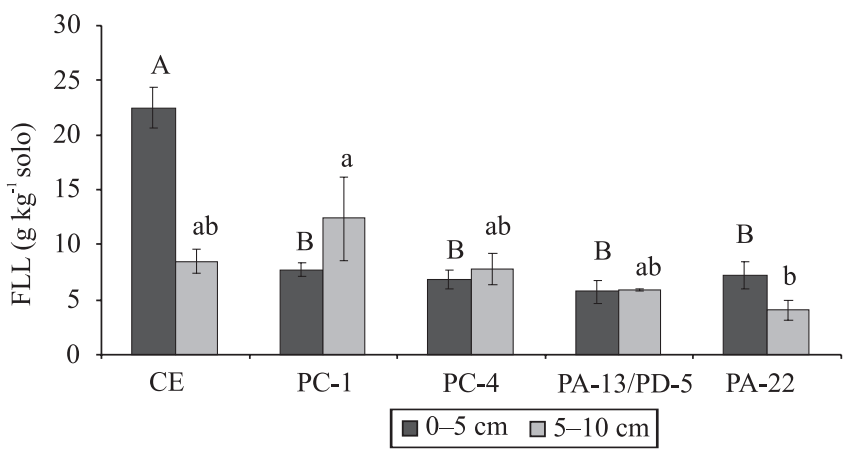

Figura 2. Distribuição do peso da fração leve livre (FLL), nas camadas 0-5 e 5-10 cm de um Neossolo Quartzarênico, sob diferentes usos agrícolas. Médias \pm erropadrão seguidas de letras iguais, maiúsculas na camada de $0-5$ cm e minúsculas na de 5-10 cm, não diferem entre si pelo teste de Tukey, a 5\% de probabilidade. CE, cerrado nativo; PC-1, soja por 1 ano (SPC); PC-4, soja por 3 anos (SPC); PA-13/ PD-5, pastagem por 13 anos seguida de soja por 5 anos (SPD); PA-22, pastagem por 22 anos. 
corretivas. Isso determina a importância da adoção de sistemas de produção que mantenham ou incrementem a MOS logo nos primeiros anos de implantação.

O solo sob cerrado nativo apresentou as maiores quantidades de fração leve livre (FLL), na camada 0-5 cm de profundidade (Figura 2). Em áreas naturais, o aporte de serrapilheira (fragmentos de folhas, galhos e raízes) é restrito à camada superficial do solo, e os estoques de $\mathrm{C}$ e $\mathrm{N}$ da FLL representam a capacidade da vegetação em manter o estoque total de $\mathrm{C}$ e $\mathrm{N}$ da $\mathrm{MOS}$ (Christensen, 1992; Rovira \& Vallejo, 2002; Rangel \& Silva, 2007).

$\mathrm{O}$ uso do solo com pastagem e culturas anuais reduziu as quantidades totais de FLL, principalmente na camada de 0-5 cm (Figura 2). Esses resultados corroboram outros obtidos em solos arenosos (Zinn et al., 2002) e argilosos (Freixo et al., 2002; Roscoe
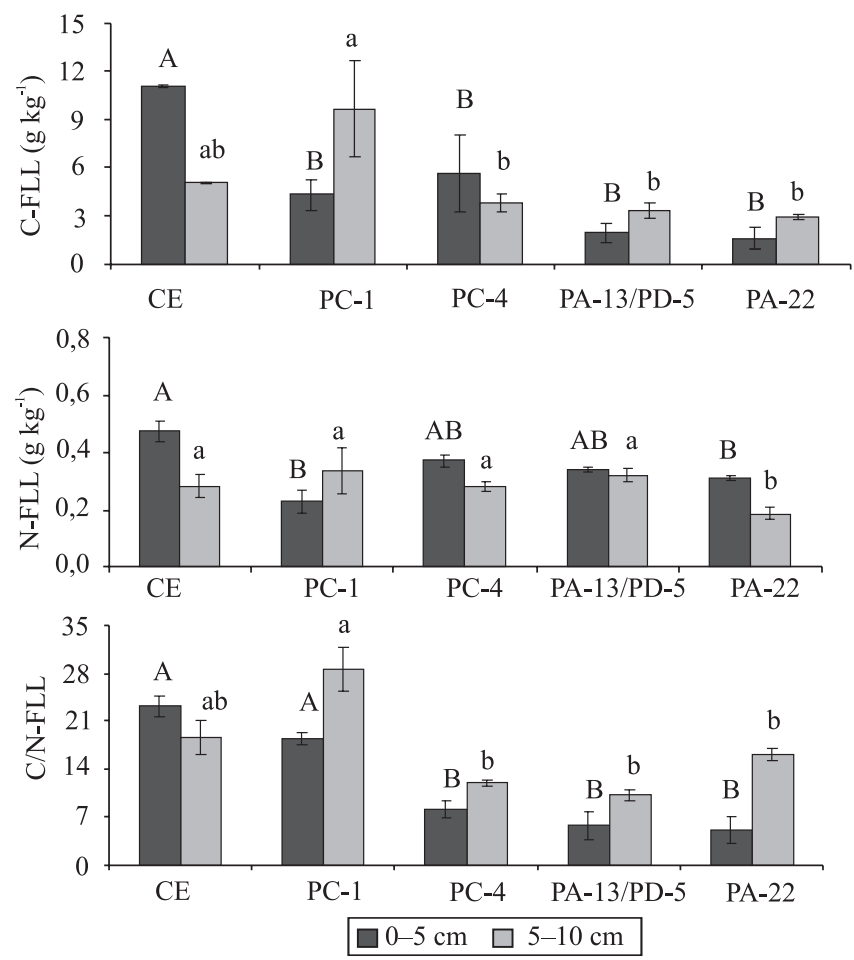

Figura 3. Teores médios de carbono $(\mathrm{C})$ e de nitrogênio $(\mathrm{N})$ e relação $\mathrm{C} / \mathrm{N}$ da fração leve livre (FLL), nas camadas 0-5 e 5-10 cm, de um Neossolo Quartzarênico sob diferentes usos agrícolas. Médias \pm erro-padrão seguidas de letras iguais, maiúsculas na camada de $0-5 \mathrm{~cm}$ e minúsculas na de 5-10 cm, não diferem entre si pelo teste de Tukey, a 5\% de probabilidade. CE, cerrado nativo; PC-1, soja por 1 ano (SPC); PC-4, soja por 3 anos (SPC); PA-13/PD-5, pastagem por 13 anos seguida de soja por 5 anos (SPD); PA-22, pastagem por 22 anos.
\& Buurman, 2003; Rangel \& Silva, 2007), que demonstram que essa fração é bastante lábil e sensível ao manejo do solo.

Cabe destacar que, tanto sob PC-1 quanto sob PC-4, as quantidades da FLL na camada $5-10 \mathrm{~cm}$ foram superiores às encontradas na camada de $0-5 \mathrm{~cm}$. Esses resultados mostram que o preparo convencional do solo promove a incorporação dos resíduos culturais e provenientes da vegetação nativa em profundidade.

Os teores de C na FLL da MOS variaram entre 16,3 e $110,0 \mathrm{~g} \mathrm{~kg}^{-1}$, e os de $\mathrm{N}$ entre 1,9 e 4,8 $\mathrm{g} \mathrm{kg}^{-1}$ (Figura 3). Como a FLL da MOS é sensível a mudanças por práticas de manejo, os maiores valores de $\mathrm{C}$ e $\mathrm{N}$ foram observados na camada $0-5 \mathrm{~cm}$ do solo, sob vegetação nativa. Na camada 5-10 cm, a área PC-1 apresentou maiores teores de $\mathrm{C}$, e a área PA-22 apresentou os menores valores de $\mathrm{N}$.

Comparando-se o solo sob uso agrícola e sob cerrado (CE), observou-se que os teores de C na FLL foram menores nas áreas PC-4, PA-13/PD-5 e PA-22. Esses resultados indicam que no cerrado ocorreu maior proteção física da FLL (Rangel \& Silva, 2007). A área de pastagem encontrava-se em estágio avançado de degradação, o que justifica os baixos estoques de $\mathrm{C}$ na FLL. As áreas sob plantio convencional (PC-4) e a convertida em sistema de plantio direto (PA-13/PD-5) apresentavam o mesmo sistema de sucessão de culturas (soja-milheto), de forma que não apresentaram diferenças significativas nos teores de $\mathrm{C}$ na FLL entre esses sistemas. Em relação ao CE, a redução dos valores de C da FLL, em PC-4 e PA-13/ PD-5, foi de 72 e $82 \%$, respectivamente, na camada 0-5 cm. Reduções dessa magnitude também foram observadas em outros estudos, que compararam diferentes sistemas de cultivo em relação a um sistema nativo (Freixo et al., 2002; Rangel \& Silva, 2007).

A relação $\mathrm{C} / \mathrm{N}$ da FLL depende do material de origem e é maior do que a relação $\mathrm{C} / \mathrm{N}$ total do solo (Paul et al., 2008). Assim, observa-se que o sistema nativo, que recebe material constantemente, e a área PC-1, instalada há pouco tempo, apresentaram as maiores relações $\mathrm{C} / \mathrm{N}$ em relação às demais áreas de estudo.

No sistema nativo, as proporções de $\mathrm{C}$ total e de $\mathrm{N}$ total do solo encontradas na FLL foram de 26 e $16 \%$, na camada de 0-10 cm, respectivamente (Tabela 2). Áreas com maior tempo de implantação (PC4, PA-13/PD5 e $\mathrm{PA} 22$ ) apresentaram valores menores de estoques de $\mathrm{C}$ 
Tabela 2. Variação do estoque de $\mathrm{C}$ e $\mathrm{N}$ da fração leve livre (FLL) na camada $0-10 \mathrm{~cm}$, em relação ao cerrado nativo, num Neossolo Quartzarênico sob diferentes usos agrícolas.

\begin{tabular}{lcccc}
\hline Sistemas $^{(1)}$ & $\begin{array}{c}\text { Estoque FLL } \\
\left(\mathrm{Mg} \mathrm{ha}^{-1}\right)\end{array}$ & \multicolumn{2}{c}{ Variação } & Fração/Total \\
\cline { 3 - 4 } & & \multicolumn{4}{c}{$\left(\mathrm{Mg} \mathrm{ha}^{-1}\right)$} & $(\%)$ & \\
\hline & \multicolumn{2}{c}{ Carbono $(\mathrm{C})$} \\
CE & $1,90 \pm 0,02$ & - & - & 26 \\
PC-1 & $1,05 \pm 0,06$ & $-0,85$ & 45 & 12 \\
PC-4 & $0,33 \pm 0,04$ & $-1,57$ & 83 & 4 \\
PA-13/PD-5 & $0,23 \pm 0,03$ & $-1,67$ & 88 & 3 \\
PA-22 & $0,18 \pm 0,02$ & $-1,72$ & 91 & 3 \\
\hline & & \multicolumn{2}{c}{ Nitrogênio (N) } & \\
CE & $0,085 \pm 0,006$ & - & - & 16 \\
PC-1 & $0,041 \pm 0,003$ & $-0,044$ & 52 & 7 \\
PC-4 & $0,034 \pm 0,002$ & $-0,051$ & 60 & 5 \\
PA-13/PD-5 & $0,028 \pm 0,001$ & $-0,057$ & 67 & 5 \\
PA-22 & $0,023 \pm 0,006$ & $-0,062$ & 73 & 4 \\
\hline
\end{tabular}

${ }^{(1)} \mathrm{CE}$, cerrado nativo; PC-1, soja por 1 ano (SPC); PC-4, soja por 3 anos (SPC); PA-13/PD-5, pastagem por 13 anos seguida de soja por 5 anos (SPD); PA-22, pastagem por 22 anos. Os valores dos estoques representam a média \pm erro-padrão $(\mathrm{n}=3)$.

e $\mathrm{N}$ da FLL, o que indica que a manutenção da MOS será comprometida em longo prazo. Todas as áreas sob uso agrícola apresentaram redução nos estoques de $\mathrm{C}$ e $\mathrm{N}$ da FLL, na profundidade de $0-10 \mathrm{~cm}$, em relação ao sistema CE, o que indica um baixo aporte de resíduos pelos sistemas agrícolas utilizados, associado à rápida decomposição (Paul et al., 2008).

Novos estudos merecem ser incentivados em solos arenosos de regiões tropicais, para que os sistemas agrícolas possam promover um adequado aporte de resíduos, com manutenção ou incremento da MOS.

\section{Conclusões}

1. As mudanças nos estoques de carbono e nitrogênio do solo, em razão da utilização de diferentes sistemas agrícolas, podem ser determinadas pela avaliação na fração leve livre da matéria orgânica do solo.

2. A adoção de sistemas agrícolas que promovam aporte elevado de resíduos é necessária para a manutenção ou incremento da matéria orgânica em Neossolo Quartzarênico.

\section{Agradecimentos}

Ao Conselho Nacional de Desenvolvimento Científico e Tecnológico, pela concessão de bolsa; à Fundação de Amparo à Pesquisa do Estado de São Paulo, pelo financiamento do trabalho; aos proprietários das Fazendas Santa Tereza e Santa Lourdes, Luis Bedusque e Julhano Souza, pela concessão das áreas de estudo e pelo apoio logístico durante a realização das coletas de amostras de solos.

\section{Referências}

BLAKE, G.R.; HARTGE, K.H. Bulk density. In: KLUTE, A. (Ed.). Methods of soil analysis. Part 1: Physical and mineralogical methods. 2.ed. Madison: American Society of Agronomy, 1986. p.363-376.

BOCHNER, J.K.; FERNANDES, M.M.; PEREIRA, M.G.; BALIEIRO, F. de C.; SANTANA, I.K. da S. Matéria orgânica e agregação de um Planossolo sob diferentes coberturas florestais. Cerne, v.14, p.46-53, 2008.

CAMBARDELlA, C.A.; ELLIOTT, E.T. Particulate soil organic-matter changes across a grassland cultivation sequence. Soil Science Society of America Journal, v.56, p.777-783, 1992.

CHRISTENSEN, B.T. Carbon in primary and secondary organomineral complexes. In: CARTER, M.R.; STEWART, B.A. (Ed.). Structure and organic matter storage in agricultural soils. Boca Raton: CRC Lewis, 1996. p.97-165.

CHRISTENSEN, B.T. Physical fractionation of soil and organic matter in primary particle size and density separates. Advances in Soil Science, v.20, p.1-90, 1992.

DIEKOW, J.; MIELNICZUK, J.; KNICKER, H.; BAYER, C.; DICK, D.P.; KÖGEL-KNABNER, I. Carbon and nitrogen stocks in physical fractions of a subtropical Acrisol as influenced by long-term no-till cropping systems and $\mathrm{N}$ fertilization. Plant and Soil, v.268, p.319-328, 2005

ELLERT, B.H.; BETTANY, J.R. Calculation of organic matter and nutrients stored in soils under contrasting management regimes. Canadian Journal of Soil Science, v.75, p.529-538, 1995.

FELLER, C.; BEARE, M.H. Physical control of soil organic matter dynamics in the tropics. Geoderma, v.79, p.69-116, 1997.

FRAZÃO, L.A.; PICCOLO, M. de C.; FEIGL, B.J.; CERRI, C.C.; CERRI, C.E.P. Propriedades químicas de um Neossolo Quartzarênico sob diferentes sistemas de manejo no Cerrado mato-grossense. Pesquisa Agropecuária Brasileira, v.43, p.641-648, 2008

FREIXO, A.A.; CANELLAS, L.P.; MACHADO, P.L.O. de A. Propriedades espectrais da matéria orgânica leve-livre e leve intra-agregados de dois Latossolos sob plantio direto e preparo convencional. Revista Brasileira de Ciência do Solo, v.26, p.445-453, 2002.

GARCIA-PAUSAS, J.; CASALS, P.; CAMARERO, L.; HUGUET, C.; SEBASTIÀ, M.-T.; THOMPSON, R.; ROMANYÀ, J. Soil organic carbon storage in mountain grasslands of the Pyrenees: effects of climate and topography. Biogeochemistry, v.82, p.279-289, 2007

GOLCHIN, A.; OADES, J.M.; SKJEMSTAD, J.O.; CLARKE, P. Study of free and occluded particulate organic matter in soils by solid state $13 \mathrm{C} \mathrm{CP/MAS} \mathrm{NMR} \mathrm{spectroscopy} \mathrm{and} \mathrm{scanning}$ 
electron microscopy. Australian Journal of Soil Research, v.32, p.285-309, 1994.

GREGORICH,E.G.; BEARE, M.H.; MCKIM, U.F.; SKJEMSTAD, J.O. Chemical and biological characteristics of physically uncomplexed organic matter. Soil Science Society of America Journal, v.70, p.975-985, 2006.

HASSINK, J. The capacity of soils to preserve organic C and $\mathrm{N}$ by their association with clay silt particles. Plant and Soil, v.191, p.77-87, 1997.

JINBO, Z.; CHANGCHUN, S.; SHENMIN, W. Dynamics of soil organic carbon and its fractions after abandonment of cultivated wetlands in Northeast China. Soil and Tillage Research, v.96, p.350-360, 2007.

MACHADO, P.L.O. de A. Fracionamento físico do solo por densidade e granulometria para a quantificação de compartimentos da matéria orgânica do solo: um procedimento para a estimativa pormenorizada do seqüestro de carbono pelo solo. Rio de Janeiro: Embrapa Solos, 2002. 6p. (Embrapa Solos. Comunicado técnico, 9).

MORAES, J.F.L. de; NEILL, C.; VOLKOFF, B.; CERRI, C.C.; MELILlO, J.; LIMA, V.C.; STEUDLER, P.A. Soil carbon and nitrogen stocks following forest conversion to pastures in the western Brazilian Amazon Basin. Acta Scientiarum, v.24, p.1369-1376, 2002.

NEEDELMAN, B.A.; WANDER, M.M.; BOLLERO, G.A.; BOAST, C.W.; SIMS, G.K.; BULLOCK, D.G. Interaction of tillage and soil texture: biologically active soil organic matter in Illinois. Soil Science Society of America Journal, v.63, p.1326-1334, 1999.

PAUL, S.; VELDKAMP, E.; FLESSA, H. Soil organic carbon in density fractions of tropical soils under forest - pasture - secondary forest land use changes. European Journal of Soil Science, v.59, p.359-371, 2008.

PEREIRA, M.G.; LOSS, A.; BEUTLER, S.J.; TORRES, J.L.R. Carbono, matéria orgânica leve e fósforo remanescente em diferentes sistemas de manejo do solo. Pesquisa Agropecuária Brasileira, v.45, p.508-514, 2010.

RANGEL, O.J.P.; SILVA, C.A. Estoques de carbono e nitrogênio e frações orgânicas de Latossolo submetido a diferentes sistemas de uso e manejo. Revista Brasileira de Ciência do Solo, v.31, p.1609-1623, 2007.

ROSCOE, R.; BUURMAN, P. Tillage effects on soil organic matter in density fractions of a Cerrado Oxisol. Soil and Tillage Research, v.70, p.107-119, 2003.

ROSCOE, R.; MACHADO, P.L.O. de A. Fracionamento físico do solo em estudos de matéria orgânica. Dourados: Embrapa Agropecuária Oeste; Rio de Janeiro: Embrapa Solos, 2002. 86p.
ROVIRA, P.; VALLEJO, V.R. Labile and recalcitrant pools of carbon and nitrogen in organic matter decomposing at different depths in soil: an acid hydrolysis approach. Geoderma, v.107, p.109-141, 2002.

SCHLESINGER, W.H. Soil organic matter: a source of atmospheric $\mathrm{CO}_{2}$. In: WOODWELL, G.M. (Ed.). The role of terrestrial vegetation in the global carbon cycle: measurement by remote sensing. New York: John Wiley, 1984. p.111-127.

SILVA, J.E. Carbon storage in clayey Oxisol cultivated pastures in the Cerrado region, Brazil. Agriculture Ecosystem and Environment, v.103, p.357-363, 2004.

SILVA. J.E.; LEMAINSKI, J.; RESCK, D.V.S. Perdas de matéria orgânica e suas relações com a capacidade de troca catiônica em solos da região de Cerrados do oeste baiano. Revista Brasileira de Ciência do Solo, v.18, p.541-547, 1994.

SIX, J.; ELLIOT, E.T.; PAUSTIAN, K.; DORAN, J.W. Aggregation and soil organic matter accumulation in cultivated and native grassland soils. Soil Science Society of America Journal, v.62, p.1367-1377, 1998.

SOHI, S.P.; MAHIEU, N.; ARAH, J.R.M.; POWLSON, D.S.; MADARI, B.; GAUNT, J.L. A procedure for isolating soil organic matter fractions suitable for modelling. Soil Science Society of America Journal, v.65, p.1121-1128, 2001.

SOLLINS, P.; HOMANN, P.; CALDWELL, B.A. Stabilization and destabilization of soil organic matter: mechanisms and controls. Geoderma, v.74, p.65-105, 1996.

STEVENSON, F.J. Humus chemistry: genesis, composition, reactions. 2.ed. New York: J. Wiley, 1994. 496p.

STEVENSON, F.J.; ELLIOT, E.T. Methodologies for assessing the quantity and quality of soil organic matter. In: COLEMAN, D.C.; OADES, J.M.; UEHARA, G. (Ed.). Dynamics of soil organic matter in tropical ecosystems. Honolulu: University of Hawaii, 1989. p.173-198.

TIESSEN, H.; STEWART, J.W.B. Particle-size fractions and their use in studies of soil organic-matter. 2. Composition effects on organic-matter composition in size fractions. Soil Science Society of America Journal, v.47, p.509-514, 1983.

TRUMBORE, S.E.; DAVIDSON, E.A.; CAMARGO, P.B.; NEPSTAD, D.C.; MARTINELLI, L.A. Bellow-ground cycling of carbon in forests and pastures of eastern Amazonia. Global Biogeochemical Cycles, v.9, p.512-528, 1995.

ZINN, Y.L.; LAL, R.; RESCK, D.V.S. Changes in soil organic carbon stocks under agriculture in Brazil. Soil and Tillage Research, v.84, p.28-40, 2005.

ZINN, Y.L.; RESCK, D.V.S.; SILVA, J.E. da. Soil organic carbon as affected by afforestation with Eucalyptus and Pinus in the Cerrado region of Brazil. Forest Ecology and Management, v.166, p.285-294, 2002.

Recebido em 7 de outubro de 2009 e aprovado em 30 de setembro de 2010 